\title{
Energy cost of walking and functional aerobic capacity during moderate intensity exercise in adults with obstructive sleep apnea: a cross-sectional study
}

Monira Aldhahi', Shipra Puri ${ }^{2}$, Vivek Jain ${ }^{3}$ and Jeffrey E. Herrick ${ }^{4^{*}}$ (D)

\begin{abstract}
Background: Autonomic dysregulation associated with obstructive sleep apnea (OSA) may limit cardiopulmonary responses to exercise, which, in turn, may impair functional aerobic capacity (FAC) and walking economy. We aimed to characterize walking economy and FAC in OSA patients compared with healthy adults (non-OSA) and examine their relationship with OSA severity (apnea-hypopnea index [AHI]).
\end{abstract}

Methods: A total of 26 adults (OSA, $n=13$; non-OSA, $n=13$ ) participated in this cross-sectional study. In this study, the participants with OSA were between the ages of 25 and 60 years, with a body mass index of $25 \mathrm{~kg} / \mathrm{m}^{2}$ to $39 \mathrm{~kg} /$ $\mathrm{m}^{2}$, and who had undergone a recent third-party sleep study with an AHI of 5 or greater. Participants completed a maximal integrated cardiopulmonary exercise test, three separate exercise bouts of constant work rate (CWR) treadmill test at $85 \%$ of anaerobic threshold (AT), and a 10-min walk test (10MWT). Multiple linear regression analysis corrected for weight, age, and BMI were performed to examine the associations.

Results: There were significant differences between OSA and non-OSA participants in $\mathrm{VO}_{2 \text { peak }}(29.7 \pm 5.6 \mathrm{~mL} / \mathrm{kg} / \mathrm{min}$ vs. $37.5 \pm 6.5 \mathrm{~mL} / \mathrm{kg} / \mathrm{min}, p=0.03)$ and $\mathrm{Net} \mathrm{VO}_{2}$ during CWR $(12.7 \pm 5 \mathrm{vs} .19 \pm 6 \mathrm{~mL} / \mathrm{kg} / \mathrm{min}, p=0.02)$. The $10 \mathrm{MWT}$ speed and distance were significantly lower in the OSA group (all $p<0.001$ ). The energy cost of walking during submaximal exercise and 10-min walk test was higher among patients with OSA (all $p<0.001$ ). The AHI scores were associated with 10MWT distance $\left(R^{2}=0.85, p<0.001\right)$, energy cost of walking $\left(R^{2}=87, p<0.001\right)$, and $\mathrm{VO}_{2}$ at anaerobic threshold $\left(R^{2}=0.92, p<0.001\right)$.

Conclusions: The findings of this study show that patients with OSA have reduced FAC and a higher energy cost of walking. AHI explained $87 \%$ of variance in the energy cost of walking during the $10 \mathrm{MWT}$. The results suggest that individuals with more severe obstructive sleep apnea experience greater impairment in functional performance.

Keywords: Oxygen consumption, Walking economy, Exercise, Energy expenditure, Apnea severity

\footnotetext{
* Correspondence: herrick_je@lynchburg.edu

${ }^{4}$ Department of Exercise Physiology, College of Health Sciences, University of Lynchburg, Lynchburg, USA

Full list of author information is available at the end of the article
}

(c) The Author(s). 2021 Open Access This article is licensed under a Creative Commons Attribution 4.0 International License, which permits use, sharing, adaptation, distribution and reproduction in any medium or format, as long as you give appropriate credit to the original author(s) and the source, provide a link to the Creative Commons licence, and indicate if changes were made. The images or other third party material in this article are included in the article's Creative Commons licence, unless indicated otherwise in a credit line to the material. If material is not included in the article's Creative Commons licence and your intended use is not permitted by statutory regulation or exceeds the permitted use, you will need to obtain permission directly from the copyright holder. To view a copy of this licence, visit http://creativecommons.org/licenses/by/4.0/ 


\section{Background}

Walking is the most common form of human physical activity that requires metabolic energy. It is the outcome of integration of multiple physiological systems working together to sustain walking pace for the required metabolic demand. Any impairment in the body system might have a catastrophic effect on walking economy and functional aerobic capacity (FAC). Meanwhile, patients with obstructive sleep apnea (OSA), a repetitive upper airway obstruction leads to abnormal nocturnal gas exchange (Guilleminault and Abad 2004), arterial oxygen desaturation, and chronically elevated sympathoadrenal activity (Carlson et al. 1993; Somers et al. 1995). Furthermore, sleep disorders have been shown to limit the physical capacity in adults (Puri et al. 2017) and is commonly associated with significant impairment in daytime physical functioning, fatigue, and daytime sleepiness that may limit daily life activities (Aguillard et al. 1998). By comparison, maximal oxygen consumption is reduced (Mendelson et al. 2018), which indicates that patients with OSA expend more energy and use a greater percentage of maximal metabolic capacity when completing activities of daily living. Despite extensive and ongoing scientific attention, understanding of the walking energy cost and mechanical efficiency for a given submaximal speed of walking in individuals with OSA have scarcely been studied.

Previous studies on OSA have reported that the presence of OSA negatively influences the aerobic capacity as measured by maximal oxygen uptake $\left(\mathrm{VO}_{2}\right.$ max) (Berger et al. 2019; Lin et al. 2006; Nanas et al. 2010; Ucok et al. 2009; Vanhecke et al. 2008; Vanuxem et al. 1997). Similarly, OSA severity is associated with an attenuated rate of oxygen consumption when calculated using a nomogram based on age, sex, baseline activity level, and high post-exercise blood pressure (Mansukhani et al. 2013). However, it remains unknown whether disease severity, as measured by the apnea hypopnea index (AHI), is an important predictor of walking economy. As shown in Fig. 1, the main hypothesis is that patients with OSA may demonstrate a decline in walking economy and consequently an increase in the energy cost of walking indirectly explain by the decline in the functional aerobic capacity.

To our knowledge, walking performance and net oxygen consumption during submaximal exercise in patients with OSA are not clearly determined. Therefore, the aims of the present study were (1) to characterize the functional determinants of walking performance and functional aerobic capacity in individuals with OSA compared with healthy individuals, and (2) to examine the relationship between OSA and walking performance outcomes in people with OSA. Quantification of physiological and functional profile of level walking and during submaximal exercise may add new and clinically meaningful perspective to the assessment of treatment outcomes following exercise interventions.

\section{Methods}

Study design and participants

In this cross-sectional study, a convenience sample of 26 volunteers was recruited from a local sleep medicine clinic and local community; 13 patients with OSA and 13 healthy adults participated in this study. Patients who met the inclusion criteria were enrolled in the study and invited to the functional performance laboratory at the George Mason University. An overnight diagnostic polysomnography (PSG) assessment was completed within 5 years of enrollment of participants in the study and was used to determine the presence of OSA and sleep

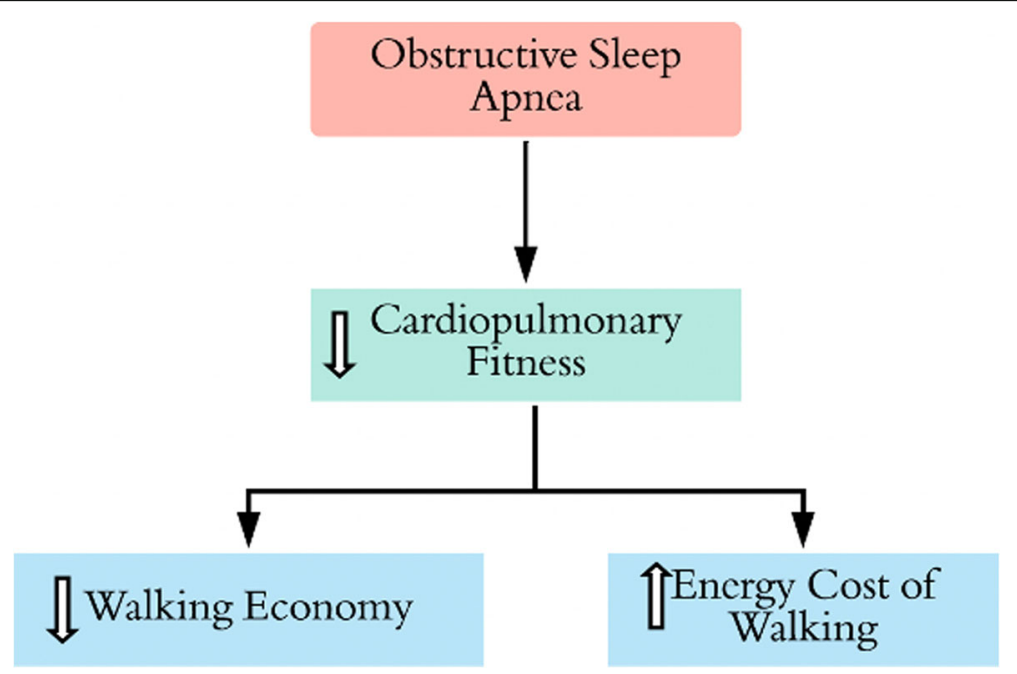

Fig. 1 Conceptual framework that illustrates the hypothetical relationship between obstructive sleep apnea and Walking performance 
parameters. The severity of OSA was determined using the apnea-hypopnea index (AHI), which measures the number of apneas and hypopneas (10 $\mathrm{s}$ or longer per event) that occur per hour of sleep.

The participants in this study were adults between the ages of 25 and 60 years, with a body mass index (BMI) between 25 and $39 \mathrm{~kg} / \mathrm{m}^{2}$, who had undergone a recent third-party sleep study with an AHI of 5 or greater; who were capable of walking on an exercise treadmill; were non-smokers; and were free of cardiovascular, metabolic, and lung disease. Participants who reported that they had been or were presently being treated by a physician for any form of cardiovascular, pulmonary, or metabolic disease and/or had any blood clotting history or vascular disease were excluded from this study. Participants were also required to answer no to all questions on the Physical Activity Readiness Questionnaire $\left(\mathrm{PARQ}^{+}\right)$and not be at high risk for a cardiac event as determined by the health history questionnaire and the American College of Sports Medicine (ACSM) risk assessment model (American College of Sports Medicine 2018).

Each study participant underwent a standard assessment of height, weight, and body mass index, as well as neck and abdominal circumference measurement, resting heart rate, and blood pressure measurement. Body composition was examined using bioimpedance analysis (Tanita Corp., Tokyo, Japan).

\section{Ethical consideration}

The research protocol was approved by the relevant institutional review boards of the George Mason University prior to initiation. Obtaining written informed consent from each participant was a condition for enrollment in the study in accordance with the Declaration of Helsinki (General Assembly of the World Medical 2014).

\section{Physical activity questionnaire}

Weekly physical activity status was determined by using the seven-day Physical Activity Recall Questionnaire (PARQ) (Hayden-Wade et al. 2003; Sallis et al. 1997). Physical activity (PA) status and total duration of PA were determined for all participants.

\section{Cardiopulmonary exercise testing}

The participants were required to visit the laboratory on two separate occasions. During the first visit, the peak oxygen consumption and anaerobic threshold (AT) were determined. Additionally, the participants underwent a maximal integrated cardiopulmonary exercise test (ICPET) on the treadmill using an individualized ramp protocol. The workload of the treadmill protocol was pre-determined on the basis of each participant's body weight and height and their self-selected comfortable walking speed (Myers et al. 1992). ICPET was performed on a Medgraphics ${ }^{\circ}$ indirect calorimetry gas analysis system $\left(\mathrm{Cardio}_{2}\right.$, St. Paul, $\left.\mathrm{MN}\right)$ for gas exchange measurements. CPET test preparation followed the guidelines of the American College of Sports Medicine (ACSM) (American College of Sports Medicine 2018). AT was determined using the following criteria: 1) the $\mathrm{V}$-slope method, defined as the inflection point from linearity of carbon dioxide output $\left(\mathrm{VCO}_{2}\right)$ against oxygen uptake $\left(\mathrm{VO}_{2}\right)$ panel; 2) point of increase in end-tidal oxygen tension $\left(\mathrm{PETO}_{2}\right)$; and 3$)$ point of increase in the ventilatory equivalent of oxygen $\left(\mathrm{VE} / \mathrm{VO}_{2}\right)$ and a concomitant reduction of end tidal carbon dioxide $\left(\mathrm{PETCO}_{2}\right)$ (Beaver et al. 1986; Marcus et al. 1971; Wasserman et al. 1973). The primary data obtained from the motorized treadmill exercise test included peak oxygen uptake $\left(\mathrm{VO}_{2 \text { peak }}\right)$ and $\mathrm{VO}_{2}$-at-anaerobic threshold (AT) from pulmonary gas exchange analysis. Heart rhythm and rate were assessed using continuous electrocardiogram (ECG) monitoring throughout the exercise testing.

\section{Constant work rate test (CWRT)}

The second visit was scheduled within 3-5 days of the first visit. Submaximal exercise included three square wave tests on a motorized treadmill at an exercise intensity identical to $85 \%$ of AT using the metabolic equation of ACSM to calculate the speed and grade. The test began with $3 \mathrm{~min}$ rest followed by performing 3 bouts of constant work rate (CWR) transition from rest to work rate at $80 \%$ of AT with each bout separated by an 8 -min active recovery at a speed of $1 \mathrm{mph}$ (Fig. 2).

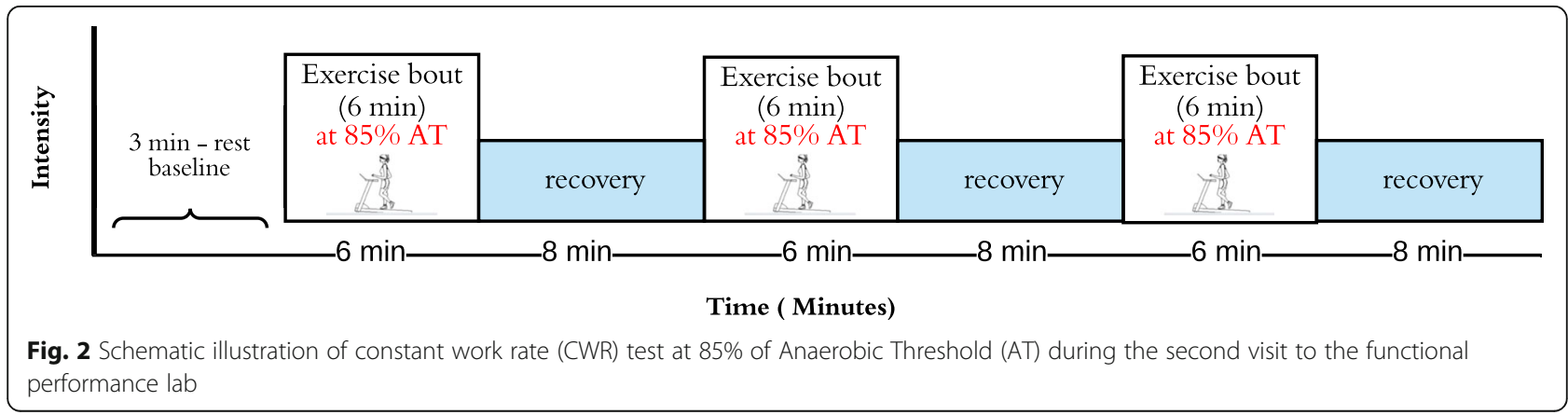


Oxygen uptake $\left(\mathrm{VO}_{2}\right)$ and walking speed at AT were determined. The net $\mathrm{VO}_{2}\left(\mathrm{VO}_{2 \text { net }}, \mathrm{mL} / \mathrm{kg} / \mathrm{min}\right)$ was calculated by subtracting the resting value of $\mathrm{VO}_{2}$ from the $\mathrm{VO}_{2}$ at a steady state work rate during treadmill exercise that corresponded to $85 \%$ of the AT $\left(\mathrm{VO}_{2 \text { net }}\right.$ $=V O_{2 \text { Exercise }}-V O_{2 \text { Resting }}$ ) (Ludlow and Weyand 2017). Steady state $\mathrm{VO}_{2}$ was calculated by averaging the last 2min $\mathrm{VO}_{2}$ values of three consecutive 6 -min bouts. The energy cost of walking during submaximal exercise corresponded to $85 \%$ of the AT.

\section{Over-ground 10-min walking test (10MWT)}

Participants were instructed to walk as far as possible around a $25-\mathrm{m}$ indoor walking track for $10 \mathrm{~min}$. The distance covered was recorded every $2.5 \mathrm{~min}$ and after 10 min. The target for this test was the completion of 10 min of walking. The participants were encouraged to complete the test without stopping. Walking performance during the 10MWT was expressed as the total distance covered in $10 \mathrm{~min}$, walking speed, and the energy cost of walking. The energy cost of over-ground walking is known as the amount of oxygen a person consumes per kilogram of body weight per distance traveled. For the present study, it was derived from the 10MWT and calculated as the amount of $\mathrm{VO}_{2}$ over the speed, where the speed was defined as the distance walked in $10 \mathrm{~min}$ $\left(\right.$ Energy cost of walking $\left.=\frac{\mathrm{VO}_{2}}{\text { Speed }}\right)$ (that is, $\mathrm{mL} \cdot \mathrm{kg}^{-1} \cdot \mathrm{m}^{-1}$ ) (Waters and Mulroy 1999).

\section{Statistical analysis}

An estimated sample size calculation was based on detecting a difference of $8.8 \mathrm{ml} / \mathrm{kg} / \mathrm{min}$ on peak oxygen consumption, a standard deviation of 6.3 and estimated for $80 \%$ power (Rizzi et al. 2013). Therefore, the estimated sample size was calculated to be at least eight subjects per group. Convenience sampling, which is a non-probability sampling, was performed during the recruitment. The participants were recruited through fliers or pamphlets in the support group or clinical center consisting of people easy to contact or reach.

For descriptive purposes, the data is presented as mean and standard deviation. After testing for normality, statistical comparisons were made between the OSA and non-OSA groups (comparison group). The indices used to determine physiological aerobic capacity were $\mathrm{VO}_{2 \text { peak }}$ and $\mathrm{VO}_{2}$-at-AT, whereas net $\mathrm{VO}_{2}$ and energy expenditure were obtained from the CWR protocol. Functional walking outcome variables were derived from the 10MWT, including gait speed over $10 \mathrm{~min}$ (meters/m) and over-ground walking energy expenditure $\left(\mathrm{mL} \cdot \mathrm{kg}^{-1} \cdot \mathrm{m}^{-1}\right)$, and distance $(\mathrm{m})$.

The baseline characteristics of the two groups were compared using an independent $t$-test. Analysis of covariance (ANCOVA) was used to determine whether significant differences in FAC, the energy cost of walking, and net $\mathrm{VO}_{2}$ emerged between the two groups, using weight, BMI, and age as covariates in the model. Multiple linear regression corrected for weight, age, and BMI was conducted to examine the associations between $\mathrm{VO}_{2 \text { peak }}$ (mL.kg ${ }^{-1}$. $\left.\mathrm{min}^{-1}\right)$, 10MWT distance (m), and energy cost of walking $\left(\mathrm{mL} \cdot \mathrm{kg}^{-1} \cdot \mathrm{min}^{-1}\right)$, treadmill energy cost of walking, and AHI. Statistical significance was set at $p \leq 0.05$. All data analyses were conducted using the STATA version 16 statistical program (StataCorp, College Station, Texas 77, 845 USA).

\section{Results}

Among 26 participants, 13 with OSA had an average AHI of $45 \pm 28$, and the mean age, weight, and BMI was $47.53 \pm 9.6$ years, $88 \pm 1 \mathrm{Kg}$, and $29 \pm 5 \mathrm{~kg} / \mathrm{m}^{2}$, respectively. The non-OSA group also consisted of 13 individuals with a mean age, weight, and BMI of $40 \pm 9$ years, $67 \pm 11 \mathrm{~kg}$, and $23 \pm 3 \mathrm{~kg} / \mathrm{m}^{2}$, respectively. A majority of OSA participants (69\%) had severe AHI, 15\% had moderate AHI, and $15 \%$ had mild AHI. Participants in both groups underwent treadmill CPET at both maximal and sub-maximal intensity of $85 \%$ of AT. Six of the 13 OSA participants were continuous positive airway pressure (CPAP) users. The baseline physical and demographic characteristics of the participants are provided in Table 1 . There were no significant differences in the baseline values between the two groups other than their weight. The physical activity level obtained through 7-PAR did not detect differences between the two groups $(p=0.64)$ (Table 1$)$.

Table 1 Demographic and physical characteristics of the study population

\begin{tabular}{llll}
\hline Variables & $\begin{array}{l}\text { OSA } \\
\boldsymbol{n}=\mathbf{1 3}\end{array}$ & $\begin{array}{l}\text { Non-OSA } \\
\boldsymbol{n}=\mathbf{1 3}\end{array}$ & $\boldsymbol{p}$-value \\
& $\begin{array}{l}\text { Mean } \pm \text { SD } \\
\text { Mean } \pm \text { SD }\end{array}$ & \\
\hline Weight (kg) & $87.62 \pm$ & $68.93 \pm$ & $0.006^{*}$ \\
& 19.4 & 11.27 & \\
Height (cm) & $172.15 \pm$ & $169.46 \pm 8.9$ & NS \\
& 6.8 & & \\
BMI (cm2/kg) & $29 \pm 5$ & $24 \pm 3$ & $0.002^{*}$ \\
Age (years) & $47.53 \pm 9.6$ & $40.46 \pm 8.8$ & NS \\
Resting HR (b/min) & $74.22 \pm 7.3$ & $77.11 \pm 14.68$ & NS \\
Gender (M: F) & $9: 4$ & $10: 3$ & NS \\
RER at VO2 peak & $1.23 \pm 0.09$ & $1.24 \pm 0.09$ & NS \\
Resting VO2(mL/kg/min) & $3.5 \pm 0.78$ & $3.85 \pm 1.35$ & NS \\
Sleep duration (Hours/Week) & $49 \pm 6$ & $51 \pm 5$ & NS \\
7-day Physical activity recall & $7.5 \pm 3.4$ & $8 \pm 3.1$ & NS \\
(METs) & & & \\
\hline
\end{tabular}

Values are expressed as Mean (SD) or frequency

Abbreviation: OSA Obstructive Sleep Apnea, $\mathrm{VO}_{2}$ oxygen uptake, $R E R$ respiratory exchange ratio, $N S$ not significant

*Denotes statistically significant $p \leq 0.05$ 
The data on maximal cardiopulmonary capacity is presented in Table 2. There were significant differences in $\mathrm{VO}_{\text {2peak }}(\mathrm{OSA} 29.69 \pm 5.6 \mathrm{~mL} / \mathrm{kg} / \mathrm{min}$ vs. non-OSA $37.49 \pm 6.5 \mathrm{~mL} / \mathrm{kg} / \mathrm{min}, p=0.03)$. Compared to the nonOSA group, the OSA participants had significantly $(P<$ $0.001)$ lower walking performance, and OSA patients tended to have higher energy cost of walking compared to the non-OSA group, as well as higher energy expenditure (Table 3). The $\mathrm{VO}_{2}$ net of the participants with OSA during CWR was significantly lower than that of the nonOSA participants after controlling for age, weight, BMI, and speed $\mathrm{m} / \mathrm{min}(13 \pm 6$ vs. $19 \pm 5, p=0.02)$. In Table 3 , the distance covered during the 10MWT was significantly lower for the OSA participants than for the non-OSA participants $(p<0.001)$. During submaximal exercise, the oxygen pulse $\mathrm{VO}_{2} / \mathrm{HR}(\mathrm{mL} \mathrm{O} /$ /beat) of OSA group was not significantly different from that of non-OSA group $(11.70 \pm 3.31$ vs. $19 \pm 5,10.56 \pm 2.85, p=0.1)$.

A majority of patients with OSA did not use CPAP (7[54]) and only 6 patients used CPAP (6[46\%]). The findings showed that there was no significant difference in the metabolic cost of walking $(p=.45)$ and peak oxygen consumption $(p=.8)$ between CPAP users and non-CPAP-users.

A multiple regression model adjusted for confounders, including age, weight, and BMI, was used to assess the association between energy cost of walking, 10MWT speed, distance, $\mathrm{VO}_{2 \text { peak }}$, and $\mathrm{AHI}$ severity. The findings showed that AHI significantly predicted the energy cost of walking $\left(F=27.6, p<0.001, R^{2}=0.87\right)$ and AHI explains $87 \%$ of variance in the energy cost of walking during the 10MWT. There was a lack of significant association between $\mathrm{VO}_{2 \text { peak }}$ and AHI severity $(P=0.4)$. The significant associations between $\mathrm{AHI}$ and walking

Table 2 Comparison of cardiopulmonary variables at peak exercise of the study population

\begin{tabular}{|c|c|c|c|}
\hline \multirow[t]{2}{*}{ Variables } & \multirow{2}{*}{$\begin{array}{l}\text { OSA } \\
(n=13) \\
\text { Mean } \pm \text { SD }\end{array}$} & $\begin{array}{l}\text { Non-OSA } \\
(n=13)\end{array}$ & \multirow[t]{2}{*}{$p$-value } \\
\hline & & Mean \pm SD & \\
\hline Time to AT (min) & $7.0 \pm 1.41$ & $8.11 \pm 2.44$ & 0.1 \\
\hline $\mathrm{VO}_{2}$ at $\mathrm{AT}(\mathrm{mL} / \mathrm{kg} / \mathrm{min})$ & $19 \pm 5$ & $25 \pm 6$ & 0.05 \\
\hline Test Duration (min) & $10.2 \pm 1.5$ & $10.8 \pm 2.7$ & 0.08 \\
\hline $\mathrm{VCO}_{2}(\mathrm{~mL} / \mathrm{min})$ & $2967 \pm 763$ & $2983 \pm 717$ & 0.3 \\
\hline $\mathrm{PETO}_{2}$ & $115 \pm 3$ & $115 \pm 6$ & 0.2 \\
\hline $\mathrm{PETCO}_{2}$ & $32 \pm 2$ & $31 \pm 4$ & 0.5 \\
\hline$H R_{\text {Peak }}(\mathrm{b} / \mathrm{min})$ & $160 \pm 13$ & $167 \pm 21$ & 0.9 \\
\hline VENO $\mathrm{NO}_{2 \text { Peak }}$ ratio (no unite) & $31 \pm 4.2$ & $37 \pm 6.3$ & 0.6 \\
\hline VENCO ${ }_{2 \text { Peak }}$ ratio (no unite) & $25 \pm 6.6$ & $29 \pm 3.6$ & 0.03 \\
\hline $\mathrm{VO}_{2} / \mathrm{HR}_{\text {peak }}\left(\mathrm{mL} \mathrm{O} \mathrm{O}_{2} /\right.$ beat $)$ & $17.92 \pm 5.4$ & $17.46 \pm 5.6$ & 0.2 \\
\hline
\end{tabular}

Value are means \pm SD

Abbreviation: AT anaerobic threshold, CWR constant work rate, $H R$ heart rate ANCVOA testing were used corrected for the age, weight, BMI
Table 3 Outcome of walking economy during submaximal exercise and 10-min walk test (10 MWT) among the study groups

\begin{tabular}{|c|c|c|c|}
\hline \multirow[t]{2}{*}{ Variables } & $\begin{array}{l}\text { OSA } \\
(n=13)\end{array}$ & $\begin{array}{l}\text { Non-OSA } \\
(n=13)\end{array}$ & \multirow[t]{2}{*}{$p$-value } \\
\hline & Mean \pm SD & Mean \pm SD & \\
\hline $10 \mathrm{MWT} \mathrm{VO}_{2}(\mathrm{~mL} / \mathrm{kg} / \mathrm{min})$ & $11.27 \pm 0.48$ & $14.45 \pm 0.80$ & $<0.001^{*}$ \\
\hline 10 MWT Speed (m/min) & $178 \pm 5$ & $110 \pm 8$ & $<0.001^{*}$ \\
\hline 10 MWT Distance (m) & $777.48 \pm 48$ & $1095.64 \pm 83$ & $<0.001^{*}$ \\
\hline $\begin{array}{l}10 \text { MWT Energy cost of } \\
\text { walking }(\mathrm{mL} / \mathrm{kg} / \mathrm{m})\end{array}$ & $0.14 \pm 0.003$ & $0.13 \pm 0.002$ & $<0.001^{*}$ \\
\hline Energy cost of CWR $(\mathrm{mL} / \mathrm{kg} / \mathrm{m})$ & $0.019 \pm 0.003$ & $0.015 \pm 0.004$ & 0.0007 \\
\hline $\mathrm{CWR} \mathrm{VO}_{2} / \mathrm{HR}$ (mL $\mathrm{O}_{2} /$ beat) & $11.70 \pm 3.31$ & $10.56 \pm 2.85$ & 0.1 \\
\hline
\end{tabular}

Value are means \pm SD

ANCVOA testing were used corrected for the age, weight, BMI

Abbreviation: $A T$ anaerobic threshold, CWR constant work rate, $H R$ heart rate

*denote statistically significant $p \leq 0.05$

speed, distance, and $\mathrm{VO}_{2}$ at the anaerobic threshold $(p<0.001)$ is presented in Table 4.

\section{Discussion}

A major finding of this study is that OSA participants had impaired walking performance and aerobic walking capacity when compared to non-OSA adults. Our results indicate that the metabolic cost of walking was high in the OSA group compared to the control group, and their maximal oxygen consumption was reduced by comparison. The severity of OSA, as reflected by AHI, was found to be associated with the rate of oxygen consumption at $\mathrm{AT}$, which is a robust predictor of aerobic exercise performance. There was a significant relationship between OSA severity (AHI) and the energy cost level of walking, walking distance, and net $\mathrm{VO}_{2}$ during the submaximal treadmill test. Collectively, these findings indicate that individuals with more severe OSA are more likely to expend more energy when completing activities of daily

Table 4 The Multiple linear regression analyses for the association between $\mathrm{VO}_{2}$ and walking performance variables during $10 \mathrm{MWT}$ and $\mathrm{AHI}$ of the study population

\begin{tabular}{lll}
\hline Variables & $\begin{array}{l}\text { AHI } \\
\mathbf{R}^{2}\end{array}$ & $\boldsymbol{p}$-value \\
\hline $\mathrm{VO}_{\text {2peak }}(\mathrm{mL} / \mathrm{kg} / \mathrm{min})$ & 0.45 & 0.4 \\
VO2-at-AT & 0.92 & $<0.001^{*}$ \\
10MWT distance $(\mathrm{m})$ & 0.85 & $<0.001^{*}$ \\
10MWT speed $(\mathrm{m} / \mathrm{min})$ & 0.85 & $<0.001^{*}$ \\
Energy cost of walking (mL/kg/min) & 0.87 & $<0.001^{*}$ \\
Treadmill energy cost of walking & 0.59 & $0.04^{*}$ \\
\hline
\end{tabular}

Value are means \pm SD

Abbreviation: $A H I$ apnea hypopnea index, VO2-at-AT oxygen consumption at anaerobic threshold

ANCVOA testing were used corrected for the age, weight, BMI

*Denotes $p$-value $\leq 0.05$ for the correlation coefficients 
living, which may increase the perception of fatigue. In this study, the lack of significant difference between OSA and non-OSA participants in ventilatory efficiency (VE/ $\mathrm{VO}_{2}$ and $\mathrm{VE} / \mathrm{CO}_{2}$ slope) and peak and submaximal oxygen pulse $\left(\mathrm{VO}_{2} / \mathrm{HR}\right.$ is used as a proxy for ventilatory and cardiac performance) suggests that the role of the cardiopulmonary response to exercise was not a limiting factor in the walking performance of participants.

Existing literature evaluating the cardiopulmonary response to exercise in OSA patients using a motor-driven treadmill reports conflicting results. In several studies, there was no difference in the maximal exercise capacity between healthy adults and OSA patients (Mortari et al. 2014; Flore et al. 2006). This contradictory finding may be attributed to selection criteria for study participants (who were newly diagnosed with OSA), the presence of comorbidities, and varying levels of OSA severity that may limit the generalizability of results (Flore et al. 2006). In line with our findings, other studies investigating the response to exercise testing of OSA patients have shown a decrease in exercise capacity (Lin et al. 2006; Nanas et al. 2010; Ucok et al. 2009; Vanhecke et al. 2008; Vanuxem et al. 1997). As expected, the value of peak oxygen consumption reported in this study was within the reference range $(29.6 \pm 6 \mathrm{~mL} / \mathrm{kg} / \mathrm{min})$ for the OSA population reported previously (Mortari et al. 2014). Systematic reviews show that reduction in $\mathrm{VO}_{2 \text { peak }}$ was larger in non-obese patients (body mass index $<30 \mathrm{~kg} / \mathrm{m}^{2}$ ), similar to our participant group (Berger et al. 2019; Mendelson et al. 2018).

There are a number of etiological factors by which OSA may appear to reduce the functional aerobic capacity and influence the energy cost of walking in people with severe obstructive sleep apnea. Sleep fragmentation and daytime somnolence are known to influence aerobic capacity, which may partially contribute to the reported decline in exercise tolerance and increase in the energy cost of walking in patients with OSA (Hong and Dimsdale 2003; Martin 1981; Mougin et al. 1991). A potential physiological mechanism that was not analyzed due to methodological limitations was decrease in maximal lactate concentration and delayed lactate elimination. This has been reported previously in OSA compared to age-matched controls, which may suggest impaired glycolytic and oxidative metabolism (Vanuxem et al. 1997). The findings of previous studies may explain the reported impairment of functional aerobic capacity of OSA patients.

The findings of exercise intolerance in our study demonstrated that the high energy cost of walking and decreased speed and distance in OSA patients may contribute to excessive daytime somnolence. A number of observational studies have revealed diminished exercise performance among OSA patients under the influence of sleep deprivation (Aguillard et al. 1998; Martin 1981; Van Helder and Radomski 1989). Extreme daytime somnolence in healthy men has been shown to reduce tolerance to tasks and increase the rating of perceived exertion during exercise testing (Temesi et al. 2013). Therefore, we cannot exclude the possibility of influence of sleep deprivation on the performance of the OSA participants in our study. Among several factors that must be considered are delayed clearance of post-exercise lactate in OSA patients and increased concentration of catecholamines. It has been reported that in OSA, the lactate threshold was attained at lower workload levels compared to age- and weight-matched controls, and an abnormal build-up of lactate was directly related to exercise capacity (Bonanni et al. 2004). These findings could be explained, at least in part, by the lower oxidative capacity of the muscle that promotes increased reliance on anaerobic metabolism in patients with OSA. Although local muscle metabolic impairment is not clearly understood, it would support both an early onset of metabolic acidosis and a subsequent increase in perceived fatigue (Keyser 2010). Vanuxem et al. (1997) reported a slow rate of lactate elimination, which could indicate a primary defect in oxidative metabolism secondary to the repeated events of nocturnal hypoxemia. Unfortunately, lactate concentration was not assessed in our study and would require further evaluation. Furthermore, repetitive cycles of hypoxemia and reoxygenation induce autonomic system instability which diminishes aerobic performance through changes in the muscle bioenergetic response (Bonanni et al. 2004; Vanuxem et al. 1997). Several studies have demonstrated structural changes in skeletal muscle fibers and bioenergetic system of the active muscle parallel to the modulation observed in chronic hypoxia conditions (Beitler et al. 2014; Sauleda et al. 2003). Abnormalities of the skeletal muscles, such as structural and bioenergetic changes in the skeletal muscle fibers (Sauleda et al. 2003), identified in OSA patients in a previous muscle biopsy study, may contribute to their lower maximal exercise capacity. Lower net $\mathrm{VO}_{2}$ during submaximal exercise may, in part, be explained by the recruitment of glycogenolytic fibers that occurs at lower levels of muscle contraction (Chwalbinska-Moneta et al. 1989). Reduced resistance to fatigue in these patients could be related to an increase in the energy cost of walking and decrease in the workload even after controlling for confounders, such as weight, BMI, and speed in the analysis.

In this study, it must be highlighted that the differences in body composition between the groups may rationalize some of the unexplained variance in walking performance and aerobic capacity. Studies in the broader literature have investigated the association between biomechanical modifications in gait characteristics and variations in body mass distribution (Ko et al. 2010; Maciejczyk et al. 2014). Walking in overweight or obese individuals may be affected by the body composition. It 
has been demonstrated that obese individuals with excess body weight exhibit shorter stride lengths, slow gait speed, and impaired aerobic capacity (Pataky et al. 2014). Further analysis in this study was performed between patients with OSA who had high BMI $(n=8)$ and patients with OSA who had normal body weight. The findings demonstrated no significant differences $(p>0.05)$ in the energy cost of walking, 10MWT speed, distance, and $\mathrm{VO}_{2 \text { peak. }}$.

Previous findings related to the association between OSA severity and physical performance are generally consistent with our results. In a study by Billings et al. (2016), low level of recreational activity such as walking was correlated with greater severity of sleep apnea, defined by using the apnea-hypopnea index, especially in male and obese individuals. Our findings suggest that the severity of OSA may contribute to some extent to an increase in the energy cost of walking and lower $\mathrm{VO}_{2}$ at CWR independent of age, weight, and BMI. This is also supported by a study by Mansukhani et al. (2013), which offers robust evidence that the severity of sleep-related breathing is associated with FAC.

Our study has a few limitations. First, the crosssectional design of our study did not allow us to establish a causal relationship. Second, some of the participants in the OSA group used CPAP, which could confound our results. However, there is conflicting evidence on the effect of long-term use of CPAP on exercise capacity. It has been previously reported that nocturnal use of CPAP improves exercise tolerance and dyspnea in obese patients with OSA (Pendharkar et al. 2011). On the contrary, other studies have reported contradictory findings that CPAP treatment did not change the peak oxygen consumption $\left(\mathrm{VO}_{2 \text { peak }}\right)$. To some extent, our findings may have been influenced by $20 \%$ of patients who were using CPAP. Despite this, differences between OSA and non-OSA participants in walking speed and mechanical efficiency were significant. However, future research should account for the influence of CPAP. Another limitation of this study was the weight of the OSA patient, as the findings of our study maybe partly influenced by the weight of the OSA, which may influence the gait characteristic in our sample. However, the difference in this study between lean and obese patients with OSA did not reveal any significant difference in the energy cost of walking, 10MWT speed, distance, and $\mathrm{VO}_{2 \text { peak }}$, which may rule out the potential effect of weight on the outcome of interest. Moreover, we accounted for visceral obesity and fat distribution in this study by using weight and BMI as confounders in the statistical analysis, yet there may be unknown confounders associated with obesity that we did not control. Additionally, PSG for sleep apnea was performed within 5 years in two of the patients with OSA and AHI was mild, and the remining were subjected to the test within 3-6 months.
The timing effect may create variability in apneahypopnea index (AHI). Nevertheless, readers should interpret the results with that limitation in mind. Finally, the low number of participants and limitation of this study to one research center may have interfered in the findings and made it difficult to extrapolate the data.

\section{Conclusions}

The main findings in this study are that patients with OSA exhibited an increased energy cost of walking and reduced functional aerobic capacity during walking compared to non-OSA adults. The OSA patients tended to show less mechanical efficiency, as evidenced by their lower net $\mathrm{VO}_{2}$ at the anaerobic threshold. Walking performance during 10MWT, presented as speed and distance covered, was lower in OSA participants, whereas the energy cost of walking was higher. This indicates that individuals with OSA tend to expend more energy to accomplish activities of daily living, which may reduce their level of physical activity and increase their perception of fatigue. The impairment of walking economy among OSA patients might take a toll on the physical function status and cardiovascular health. The study of movement economy and FAC response in patients with OSA is physiologically relevant to understand the adaptive changes that occur in response to hypoxia-reoxygenation episodes during sleep. Future studies need to elucidate the mechanisms involved in the response of walking economy to various targeted interventions. Moreover, investigative approaches using muscle biopsy or infrared spectroscopy (NIRS) could profoundly characterize muscle oxidative capacity and functional aspects of the skeletal muscle in this context and shed light on impaired exercise tolerance in OSA.

\section{Abbreviations \\ AHI: Apnea-hypopnea index; AT: Anaerobic test; BMl: Body mass index; CPAP: Continuous positive airway pressure; CWR: Constant work rate; ECG: Electrocardiogram; FAC: Functional aerobic capacity; ICPET: Integrated cardiopulmonary exercise test; OSA: Obstructive sleep apnea; 10MWT: 10-min walking test; PSG: Polysomnography; $\mathrm{VO}_{2}$ max: Maximal oxygen uptake \\ Acknowledgments \\ A special thank for the Deanship of Scientific Research at Princess Nourah Bint Abdulrahman University for funding this project through the Fast-track Research Funding Program, Riyadh, Saudi Arabia.}

\section{Authors' contributions}

All authors contributed substantially to the manuscript. MA, SP, and JH contributed to the conception and design of the study (MA, SP, JH). MA and SP are conducted experiments, acquired the data, and recruitment. Conceived and analyzed data (MA) Drafting the manuscript (MA, SP, JH, VJ). All authors contributed to the preparation of the manuscript and reviewed the manuscript for important intellectual content. The author(s) read and approved the final manuscript.

\section{Funding}

This research was funded by the Deanship of Scientific Research at Princess Nourah Bint Abdulrahman University through the Fast-track Research Funding Program. 


\section{Availability of data and materials}

The identified datasets analyzed during the current study are available from the corresponding author on reasonable request.

\section{Ethics approval and consent to participate}

All procedures and the research protocol in this study involving human participants were approved in accordance with the ethical standards of the Institutional Review Boards of George Mason University prior to initiation. Informed consent to participate was obtained from each participant prior to study enrollment. Obtaining written informed consent from each participant was a condition of enrollment in the study in accordance with the Declaration of Helsinki (General Assembly of the World Medical 2014).

\section{Consent for publication}

Not Applicable.

\section{Competing interests}

The authors declare that they have no competing interests.

\section{Author details}

'Department of Rehabilitation Sciences, College of Health and Rehabilitation Sciences, Princess Nourah bint Abdulrahman University, Riyadh, Kingdom of Saudi Arabia. ${ }^{2}$ Department of Physiology, School of Medicine, Wayne State University, Detroit, USA. ${ }^{3}$ Division of Pulmonary, Critical Care \& Sleep Medicine, George Washington University, Washington, USA. ${ }^{4}$ Department of Exercise Physiology, College of Health Sciences, University of Lynchburg, Lynchburg, USA.

\section{Received: 10 October 2020 Accepted: 8 February 2021} Published online: 14 April 2021

\section{References}

Aguillard RN, Riedel BW, Lichstein KL, Grieve FG, Johnson CT, Noe SL. Daytime functioning in obstructive sleep apnea patients: exercise tolerance, subjective fatigue, and sleepiness. Appl Psychophysiol Biofeedback. 1998;23(4):207-17.

American College of Sports Medicine. ACSM's guidelines for exercise testing and prescription. Philadelphia: Wolters Kluwer; 2018.

Beaver WL, Wasserman K, Whipp BJ. A new method for detecting anaerobic threshold by gas exchange. J Appl Physiol. 1986;60(6):2020-7.

Beitler JR, Awad KM, Bakker JP, Edwards BA, DeYoung P, Djonlagic I, et al. Obstructive sleep apnea is associated with impaired exercise capacity: a cross-sectional study. J Clin Sleep Med. 2014;10(11):1199-204.

Berger M, Kline CE, Cepeda FX, Rizzi CF, Chapelle C, Laporte S, et al. Does obstructive sleep apnea affect exercise capacity and the hemodynamic response to exercise? An individual patient data and aggregate metaanalysis. Sleep Med Rev. 2019;45:42-53.

Billings ME, Johnson DA, Simonelli G, Moore K, Patel SR, Roux AVD, et al. Neighborhood walking environment and activity level are associated with OSA: the Multi-Ethnic Study of Atherosclerosis. Chest. 2016;150(5):1042-9.

Bonanni E, Pasquali L, Manca ML, Maestri M, Prontera C, Fabbrini M, et al. Lactate production and catecholamine profile during aerobic exercise in normotensive OSAS patients. Sleep Med. 2004;5(2):137-45.

Carlson JT, Hedner J, Elam M, Ejnell H, Sellgren J, Wallin BG. Augmented resting sympathetic activity in awake patients with obstructive sleep apnea. Chest. 1993;103(6):1763-8.

Chwalbinska-Moneta J, Robergs RA, Costill DL, Fink WJ. Threshold for muscle lactate accumulation during progressive exercise. J Appl Physiol. 1989;66(6): 2710-6.

Flore P, Tonini J, Pepin J-L, Levy P, Wuyam B. Obstructive sleep apnoea: hypoapnoea syndrome reversibly depresses cardiac response to exercise. Eur Heart J. 2006;27:1632-3.

General Assembly of the World Medical. World Medical Association Declaration of Helsinki: ethical principles for medical research involving human subjects. J Am Coll Dent. 2014;81:14.

Guilleminault C, Abad VC. Obstructive sleep apnea syndromes. Med Clin North Am. 2004;88:611-30.

Hayden-Wade HA, Coleman KJ, Sallis JF, Armstrong C. Validation of the telephone and in-person interview versions of the 7-day PAR. (physical activity recall). Med Sci Sports Exerc. 2003;35:801-9. https://doi.org/10.1249/ 01.MSS.0000064941.43869.4E.
Hong S, Dimsdale JE. Physical activity and perception of energy and fatigue in obstructive sleep apnea. Med Sci Sports Exerc. 2003;35(7):1088-92.

Keyser RE. Peripheral fatigue: high-energy phosphates and hydrogen ions. PM\&R. 2010;2(5):347-58.

Ko S-u, Stenholm S, Ferrucci L. Characteristic gait patterns in older adults with obesity - results from the Baltimore longitudinal study of aging. J Biomech. 2010;43(6):1104-10.

Lin C-C, Hsieh W-Y, Chou C-S, Liaw S-F. Cardiopulmonary exercise testing in obstructive sleep apnea syndrome. Respir Physiol Neurobiol. 2006;150(1): 27-34.

Ludlow LW, Weyand PG. Walking economy is predictably determined by speed, grade, and gravitational load. J Appl Physiol. 2017;123(5):1288-302.

Maciejczyk M, Więcek M, Szymura J, Szyguła Z, Wiecha S, Cempla J. The influence of increased body fat or lean body mass on aerobic performance. PLoS One. 2014;9(4):e95797.

Mansukhani MP, Allison TG, Lopez-Jimenez F, Somers VK, Caples SM. Functional aerobic capacity in patients with sleep-disordered breathing. Am J Cardiol. 2013;111(11):1650-4.

Marcus JH, Ingram RH Jr, McLean RL. The threshold of anaerobic metabolism in chronic obstructive pulmonary disease: a promising index of evaluation. Am Rev Respir Dis. 1971:104(4):490-8.

Martin BJ. Effect of sleep deprivation on tolerance of prolonged exercise. Eur J Appl Physiol. 1981:47(4):345-54.

Mendelson M, Marillier M, Bailly S, Flore P, Borel J-C, Vivodtzev I, et al. Maximal exercise capacity in patients with obstructive sleep apnoea syndrome: a systematic review and meta-analysis. Eur Respir J. 2018;51(6). https://doi.org/1 0.1183/13993003.02697-2017.

Mortari D, Leguisamo CP, Fagondes SC. Exercise capacity in patients with obstructive sleep apnea syndrome. Rev Fac Ciênc Médicas Sorocaba. 2014;16:164-9.

Mougin F, Simon-Rigaud ML, Davenne D, Renaud A, Garnier A, Kantelip JP, et al. Effects of sleep disturbances on subsequent physical performance. Eur J Appl Physiol. 1991;63(2):77-82.

Myers J, Buchanan N, Smith D, Neutel J, Bowes E, Walsh D, et al. Individualized ramp treadmill: observations on a new protocol. Chest. 1992;101(5):236S-41S.

Nanas S, Sakellariou D, Kapsimalakou S, Dimopoulos S, Tassiou A, Tasoulis A, et al. Heart rate recovery and oxygen kinetics after exercise in obstructive sleep apnea syndrome. Clin Cardiol. 2010;33(1):46-51.

Pataky Z, Armand S, Müller-Pinget S, Golay A, Allet L. Effects of obesity on functional capacity. Obesity. 2014;22(1):56-62.

Pendharkar SR, Tsai WH, Eves ND, Ford GT, Davidson WJ. CPAP increases exercise tolerance in obese subjects with obstructive sleep apnea. Respir Med. 2011; 105(10):1565-71.

Puri S, Herrick JE, Collins JP, Aldhahi M, Baattaiah B. Physical functioning and risk for sleep disorders in US adults: results from the National Health and nutrition examination survey 2005-2014. Public Health. 2017:152:123-8.

Rizzi CF, Cintra F, Mello-Fujita L, Rios LF, Mendonca ET, Feres MC, et al. Does obstructive sleep apnea impair the cardiopulmonary response to exercise? Sleep. 2013;36(4):547-53.

Sallis JF, Buono MJ, Roby JJ, Micale FG, Nelson JA. Seven-day physical activity recall. Med Sci Sports Exerc. 1997;29:89-103.

Sauleda J, García-Palmer FJ, Tarraga S, Maimó A, Palou A, Agustí AG. Skeletal muscle changes in patients with obstructive sleep apnoea syndrome. Respir Med. 2003:97(7):804-10.

Somers VK, Dyken ME, Clary MP, Abboud FM. Sympathetic neural mechanisms in obstructive sleep apnea. J Clin Invest. 1995;96(4):1897-904.

Temesi J, Arnal PJ, Davranche K, Bonnefoy R, Levy P, Verges S, et al. Does central fatigue explain reduced cycling after complete sleep deprivation? Med Sci Sports Exerc. 2013;45(12):2243-53.

Ucok K, Aycicek A, Sezer M, Genc A, Akkaya M, Caglar V, et al. Aerobic and anaerobic exercise capacities in obstructive sleep apnea and associations with subcutaneous fat distributions. Lung. 2009;187(1):29-36.

Van Helder T, Radomski MW. Sleep deprivation and the effect on exercise performance. Sports Med. 1989;7(4):235-47.

Vanhecke TE, Franklin BA, Ajluni SC, Sangal RB, McCullough PA. Cardiorespiratory fitness and sleep-related breathing disorders. Expert Rev Cardiovasc Ther. 2008;6(5):745-58

Vanuxem D, Badier M, Guillot C, Delpierre S, Jahjah F, Vanuxem P. Impairment of muscle energy metabolism in patients with sleep apnoea syndrome. Respir Med. 1997;91(9):551-7. 
Wasserman K, Whipp BJ, Koyl SN, Beaver WL. Anaerobic threshold and respiratory gas exchange during exercise. J Appl Physiol. 1973;35(2): 236-43.

Waters RL, Mulroy S. The energy expenditure of normal and pathologic gait. Gait Posture. 1999;9(3):207-31.

\section{Publisher's Note}

Springer Nature remains neutral with regard to jurisdictional claims in published maps and institutional affiliations.

Ready to submit your research? Choose BMC and benefit from:

- fast, convenient online submission

- thorough peer review by experienced researchers in your field

- rapid publication on acceptance

- support for research data, including large and complex data types

- gold Open Access which fosters wider collaboration and increased citations

- maximum visibility for your research: over $100 \mathrm{M}$ website views per year

At BMC, research is always in progress.

Learn more biomedcentral.com/submissions 